
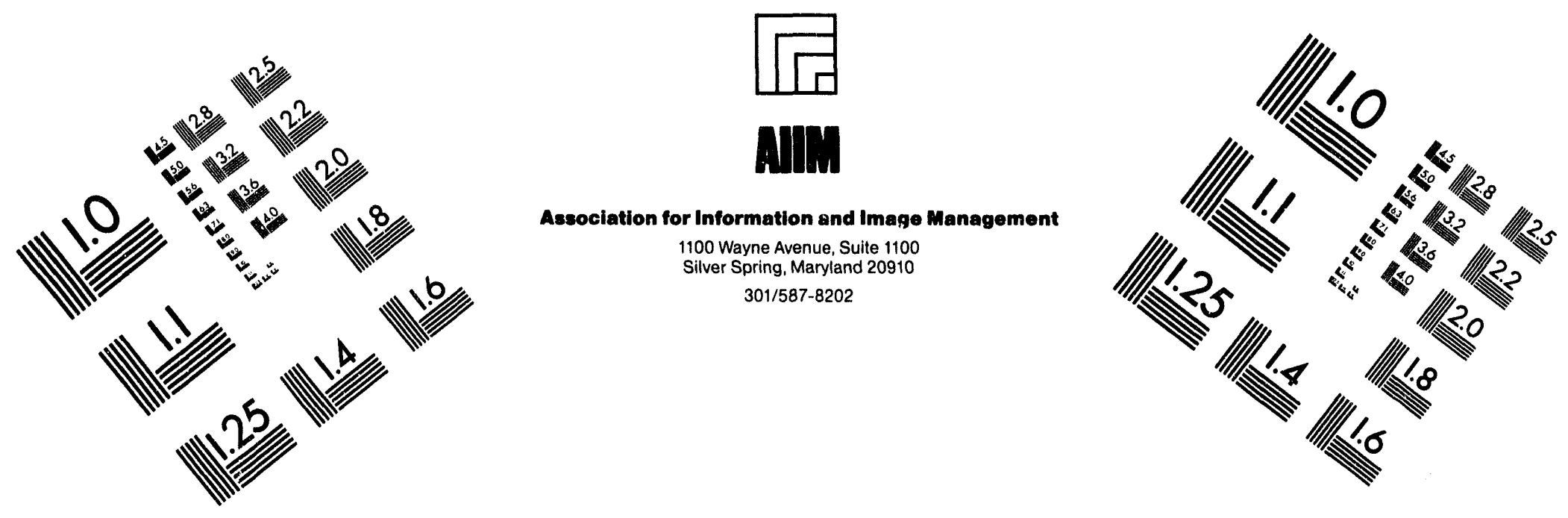

\title{
Centimeter
}

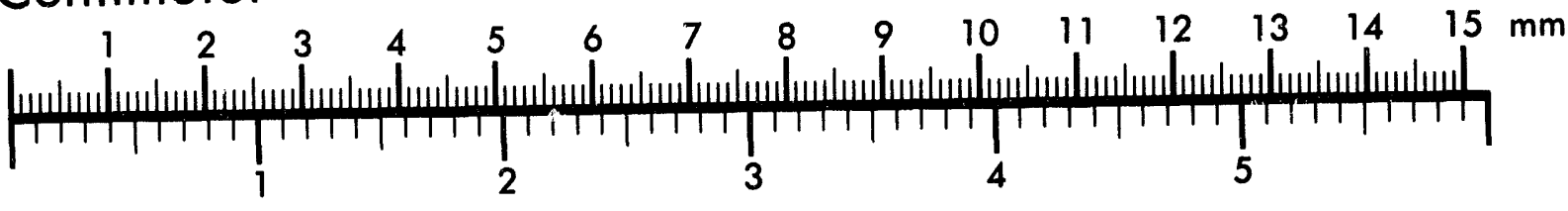
Inches
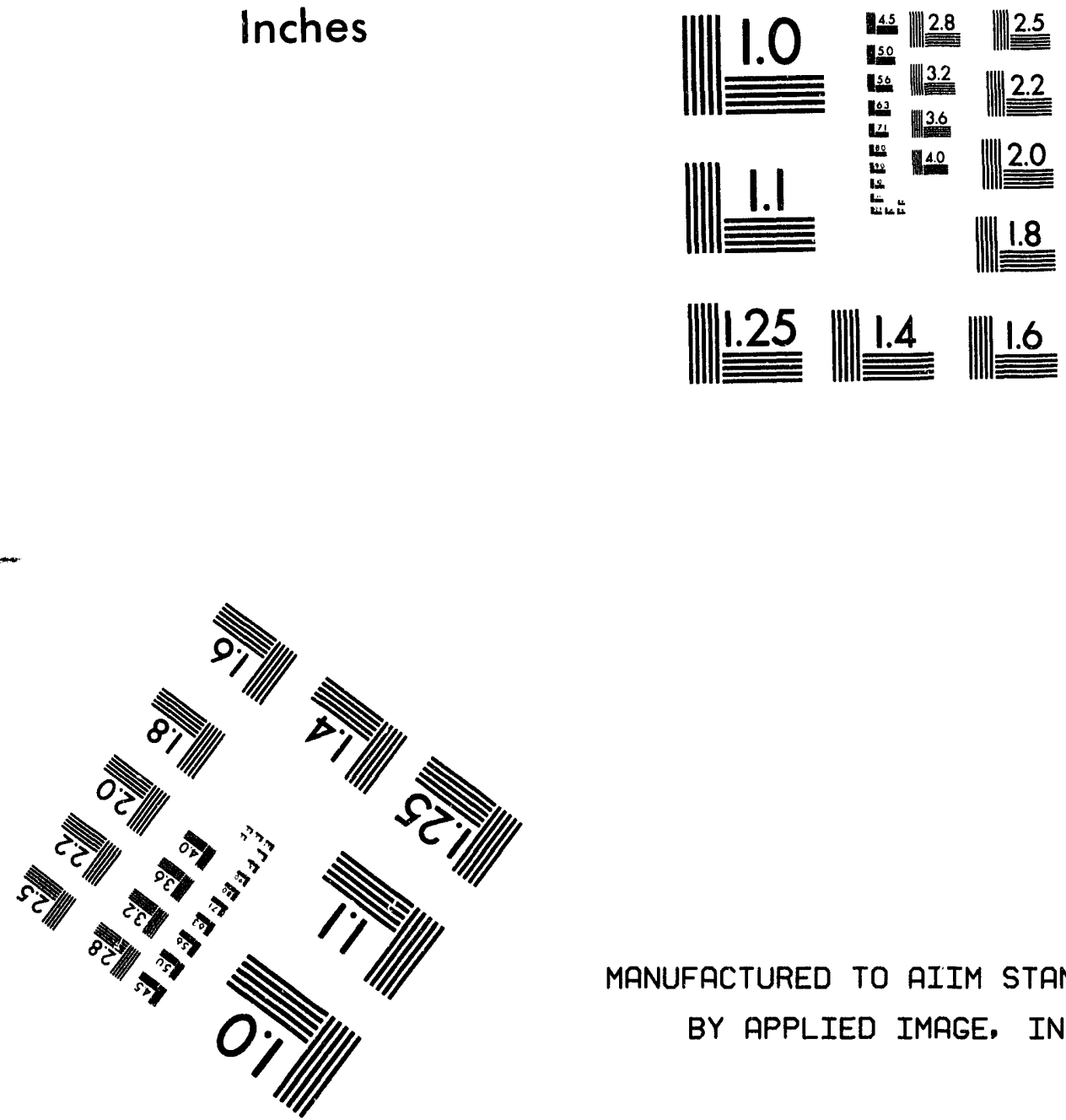

MANUFACTURED TO AIIM STANDARDS

BY APPLIED IMAGE, INC.

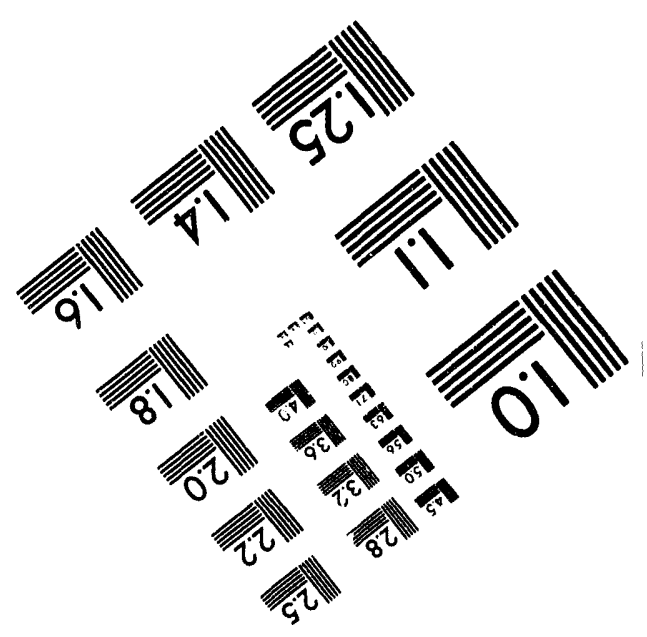



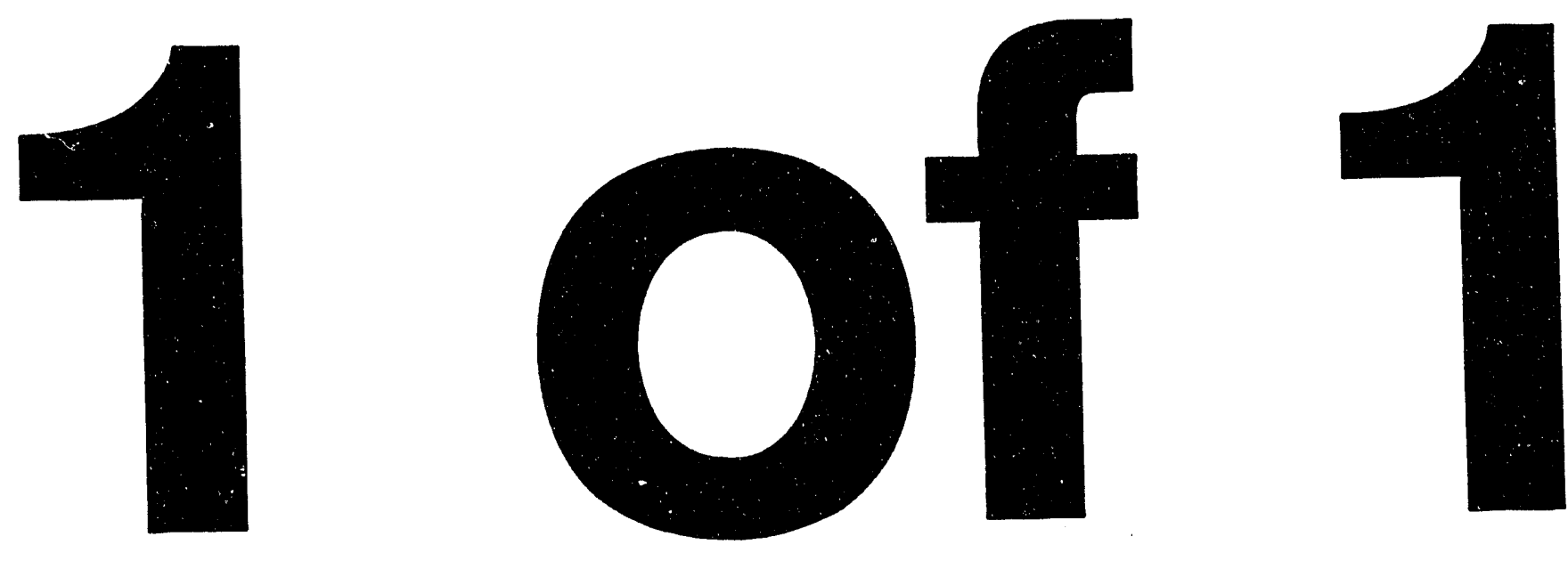


\title{
Corf- $940226--8$
}

UCRL-JC-115149

PREPRINT

\section{New Ytterbium-doped Apatite Crystals for Flexible Laser Design}

\author{
Stephen A. Payne \\ Laura D. DeLoach \\ Larry K. Smith \\ William F. Krupke \\ Lawrence Livermore National Laboratory
}

Bruce H. T. Chai

George Loutts

Center for Research and Education in Optics and Lasers

University of Central Florida

This paper was prepared for submittal to the

Optical Society of America Proceedings

on Advanced Solid-State Lasers ('94)

Salt Lake City, Utah

February 7-10, 1994

\section{March 1994}

This is a preprint of a paper intended for publication in a joumal or proceedinga. Since changes may be made before publication, this preprint is made available with the understanding that it will not be cited or reproduced without the permisoion of the author.

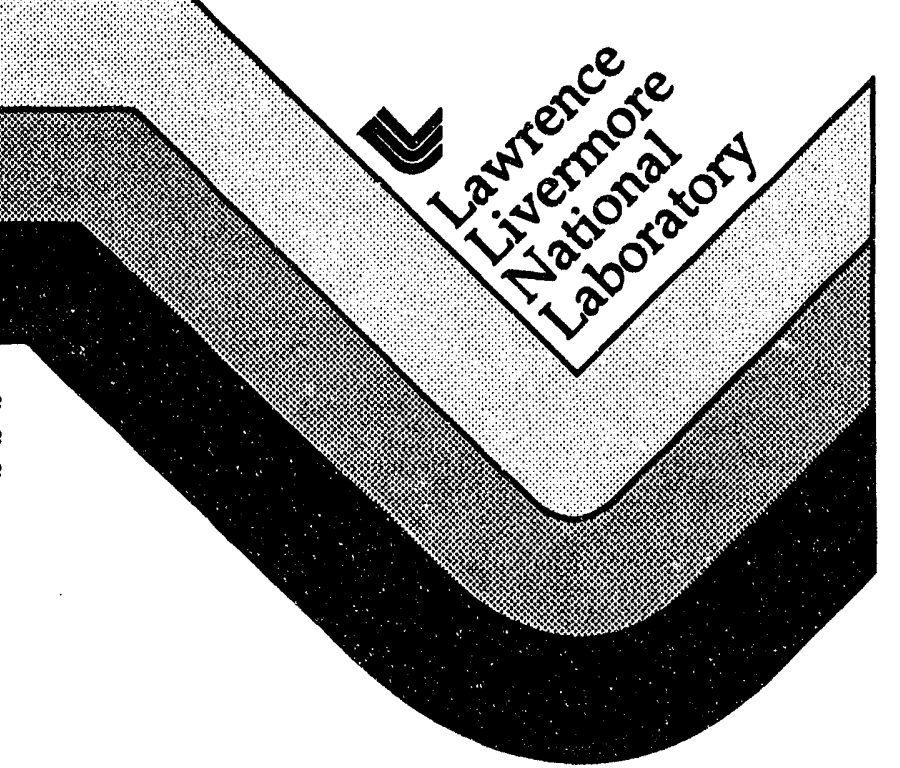

\section{MASTER}




\section{DISCLAIMER}

This document was prepared as an account of work sponsored by an agency of the United States Government. Neither the United States Government nor the University of California nor any of their employees, makes any warranty, express or implied, or assumes anylegal liability or responsibility for the accuracy, completeness, or usefulness of any information, apparatus, product, or process disclosed, or represents that its use would not infringe privately owned rights. Reference herein to any specific commercial products, process, or service by trade name, trademark, manufacturer, or otherwise, does not necessarily constitute or imply its endorsement, recommendation, or favoring by the United States Govemment or the University of California. The views and opinions of authors expressed herein do not necessarily state or reflect those of the United States Government or the University of California, and shall not be used for advertising or product endorsement purposes. 
New Ytterbium-doped Apatite Crystals for Flexible Laser Design

Stephen A. Fuy ne, Laura D. DeLoach, Larry K. Smith, and William F. Krupke
Lawrence Livermore National Laboratory, University of California P.O. Box 5508, Livermore, CA 94550

\author{
Bruce H. T. Chai and George Loutts \\ Center for Research and Education in Optics and Lasers \\ University of Central Florida, Orlando, FL 32826
}

\begin{abstract}
A new class of Yb-lasers is summarized in this article. The apatite family of crystals has been found to impose favorable spectroscopic and laser properties on the $\mathrm{Yb}^{3+}$ activator ion. Crystals of $\mathrm{Yb}$-doped $\mathrm{Ca} 5\left(\mathrm{PO}_{4}\right)_{3} \mathrm{~F}, \mathrm{Sr}_{5}\left(\mathrm{PO}_{4}\right)_{3} \mathrm{~F}$, $\mathrm{Ca}_{x} \mathrm{Sr}_{5-\mathrm{x}}\left(\mathrm{PO}_{4}\right)_{3} \mathrm{~F}$, and $\mathrm{Sr}_{5}\left(\mathrm{VO}_{4}\right)_{3} \mathrm{~F}$ have been grown and investigated. Several useful laser crystals have been identified which offer a variety of fundamental laser parameters for designing diode-pumped systems. In general, this class of materials is characterized by high emission cross sections $\left(3.6-13.1 \times 10^{-20} \mathrm{~cm}^{2}\right)$, useful emission lifetimes (0.59 - $1.26 \mathrm{msec})$, a strong pump band $\left(\sigma_{\mathrm{abs}}=2.0-10.0 \times 10^{-20} \mathrm{~cm}^{2}\right)$ and pump and extraction wavelengths near 900 and $1045 \mathrm{~nm}$, respectively. Efficient lasing has been demonstrated for several of the members of this class of materials, and high optical quality crystals have been grown by the Czochralski method.
\end{abstract}

\section{INTRODUCTION}

$\mathrm{Yb}^{3+}$ has long been recognized to serve as a laser ion when incorporated as a dopant into $\mathrm{Y}_{3} \mathrm{Al}_{5} \mathrm{O}_{12}$ and other garnets. ${ }^{1}$ The main obstacle to further development entailed the limited pump absorption features that are available with $\mathrm{Yb}$-doped crystals. Since $\mathrm{Yb}^{3+}$ is a $4 \mathrm{f}^{13}$ ion, it possesses only two relevant electronic states the ${ }^{2} \mathrm{~F}_{7 / 2}$ ground state and the ${ }^{2} \mathrm{~F}_{5 / 2}$ excited state - separated by about $10,000 \mathrm{~cm}^{-1}$. Since the prevailing pump sources in the 1960s and 1970 s only included broad white light generating flashlamps, the single narrow absorption feature of $\mathrm{Yb}^{3+}$ proved to be an inadequate means of effectively pumping the laser material. While the need for a useful narrow band pump source was recognized some time ago, ${ }^{2}$ it was not until the emergence of InGaAs laser diodes that some worker's recognized the renewed significance of $\mathrm{Yb}$ doped materials. ${ }^{3}$ In fact, with the availability of commercial laser diodes, the limited absorption features of $\mathrm{Yb}$ actually provide an advantage, since the lack of higher-lying excited states assures the absence of detrimental processes such as upconversion and excited state absorption. Additionally, the $\mathrm{Yb}^{3+}$ dopant offers an important advantage over the most common laser ion, $\mathrm{Nd}^{3+}$, since its emission lifetime tends to be about four times greater when the same host medium is considered for both ions, thereby allowing for an enhanced level of energy storage.

The main complication associated with solid-state lasers based on the $\mathrm{Yb}$ ion involves the quasi-four-level nature of the system at room temperature. Otherwise stated, the problem is that the terminal laser level tends to reside at an energy of $200-600 \mathrm{~cm}^{-1}$, which is comparable to the thermal energy present at room temperature $\left(200 \mathrm{~cm}^{-1}\right) .4$ Accordingly, the search for new $\mathrm{Yb}^{3}+$ lasers has been intimately tied to the structure of the energy levels and the strength of the transitions among them. In particular, the nature of the crystal field splitting in the ${ }^{2} \mathrm{~F} 7 / 2$ and ${ }^{2} \mathrm{~F}_{5} / 2$ states anc the cross section associated with the various ${ }^{2} \mathrm{~F}_{7 / 2}-{ }^{2} \mathrm{~F}_{5 / 2}$ transitions turns out to have a crucial impact on the viability of the system as a laser material. In brief, the ideal $\mathrm{Yb}$-laser would possess a high-lying crystal field component in the ${ }^{2} F_{7} / 2$ ground state, to which a particularly intense transition from the ${ }^{2} \mathrm{~F}_{5} / 2$ excited state would exist. The Yb-doped apatite class of laser materials to be described in this article is characterized by just such an energy level structure along with the desired transition strength moments. 5

As a result of our recent efforts, the $\mathrm{Yb}^{3+}$. doped apatite-structure crystals may now potentially be employed in a variety of diodepumped laser systems. This family of laser materials offers a substantial range of fundamental laser parameters with which systems may be designed, in order to adjust the pumping, storage, and energy extraction characteristics. In other words, the Yb-doped 
apatite class of materials allows a number of trade-offs, wherein the emission lifetime and cross section, as well as the pump line width and saturation parameters can be altered, albeit not independently of each other. A range of possibilities nuw exists such that the specific parameters of a given Yb:apatite laser medium can be matched to the requirements of a particular application. Among the crystals that have turned out to be useful $\mathrm{Yb}$ laser materials are $\mathrm{Ca}_{5}\left(\mathrm{PO}_{4}\right)_{3} \mathrm{~F}$ (FAP), ${ }^{5} \mathrm{Sr}_{5}\left(\mathrm{PO}_{4}\right)_{3} \mathrm{~F}$ (S-FAP), ${ }^{6}$ $\mathrm{Ca}_{3} \mathrm{Sr}_{2}\left(\mathrm{PO}_{4}\right)_{3} \mathrm{~F}\left(\mathrm{C}_{3} \mathrm{~S}_{2}-\mathrm{FAP}\right)_{,}{ }^{7}$ and $\mathrm{Sr}_{5}\left(\mathrm{VO}_{4}\right)_{3} \mathrm{~F}$ (S-VAP). 8 It is important to note that the apatite crystals were originally investigated as $\mathrm{Nd}$ laser host media; ${ }^{9}$ our contribution was to recognize that the $\mathrm{Yb}$ ion exhibits particularly useful laser properties in these crystals. It is also noteworthy that $\mathrm{Yb}: Y A G$ has also proved to be a highly effective diode-pumped laser material, as discussed recently. 10

\section{SPECTROSCOPY}

The measured absorption and emissic 1 crosi sections, $\sigma_{\mathrm{abs}}$ and $\sigma_{\mathrm{em}}$, and the upper level lifetimes, $\tau_{e m}$, can be used to assess several important laser performance parameters for different $Y b$ apatite lasers. In particular, $\beta_{\text {min }}$, which is defined as the minimum fraction of $\mathrm{Yb}$ ions that must be excited in order to balance the gain and the ground state absorption at the extraction wavelength, is crucial in quasi-fourlevel systems such as $\mathrm{Yb}^{3+}$. The $\beta_{\min }$ parameter may be determined then from the absorption and emission cross section values for the extraction wavelength of the laser, $\lambda$ ext, according to. $3-5$

$$
\beta_{\min }=\frac{\sigma_{\text {abs }}\left(\lambda_{\text {ext }}\right)}{\sigma_{\text {abs }}\left(\lambda_{\text {ext }}\right)+\sigma_{\text {em }}\left(\lambda_{\text {ext }}\right)} .
$$

The $\boldsymbol{\beta}_{\min }$ parameter may also be expressed in terms of the individual transitions between the crystal field components of the electronic states. $3-5$

Another performance parameter which can be assessed from the spectroscopic properties is the pump saturation intensity, Isat. For efficient diode pumping, a large fraction of $\mathrm{Yb}$ ions must be excited in order to overcome the ground state absorption losses, and Isat is a measure of the ease of bleaching the Yb-doped crystal. This pump parameter is as sessed from the $\sigma_{a b s}$ value at the pump wavelength, $\lambda_{p}$, and the emission lifetime, $\tau_{\mathrm{em}}$ using:

$$
I_{\text {sat }}=\frac{h c}{\lambda_{\mathrm{p}} \sigma_{\mathrm{abs}} \tau_{\mathrm{em}}} .
$$

Clearly, higher absorption at the pump wavelength and longer $\mathrm{Yb}$ lifetimes are desirable for low values of $I_{\text {sat. }}$.

Finally, an important parameter which takes into account both the absorption and emission characteristics of the Yb-laser crystal is $I_{\min }$. In the case of lightly-doped crystals, I min is the minimum absorbed pump intensity which is required to reach threshold (in an otherwise lossless oscillator). This parameter is simply the product of our two previous laser performance indicators or:

$$
I_{\min }=\beta_{\min } I_{\text {sat }} \text {. }
$$

Lower values of $I_{\min }$ imply lower threshold lasers and therefore are more desirable when all else considered is equal.
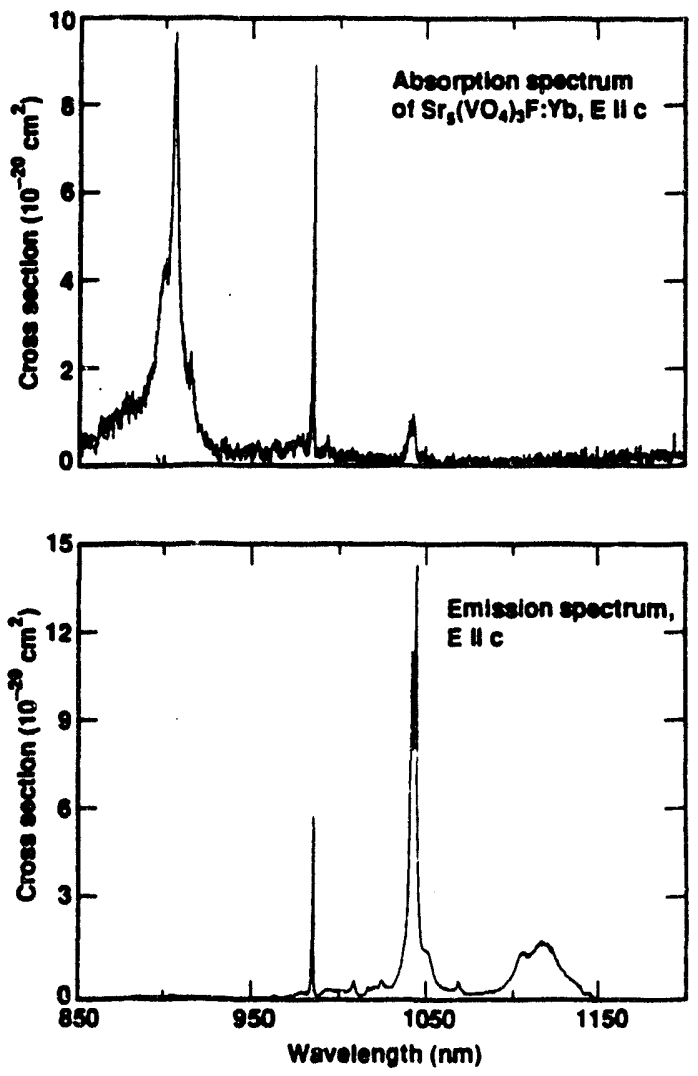

Figure 1. Absorption and emission spectra of $\mathrm{Yb}$ in $\mathrm{Sr}_{5}\left(\mathrm{VO}_{4}\right)_{3} \mathrm{~F}$ (S-VAP), with E\|c, on absolute cross section scale. 

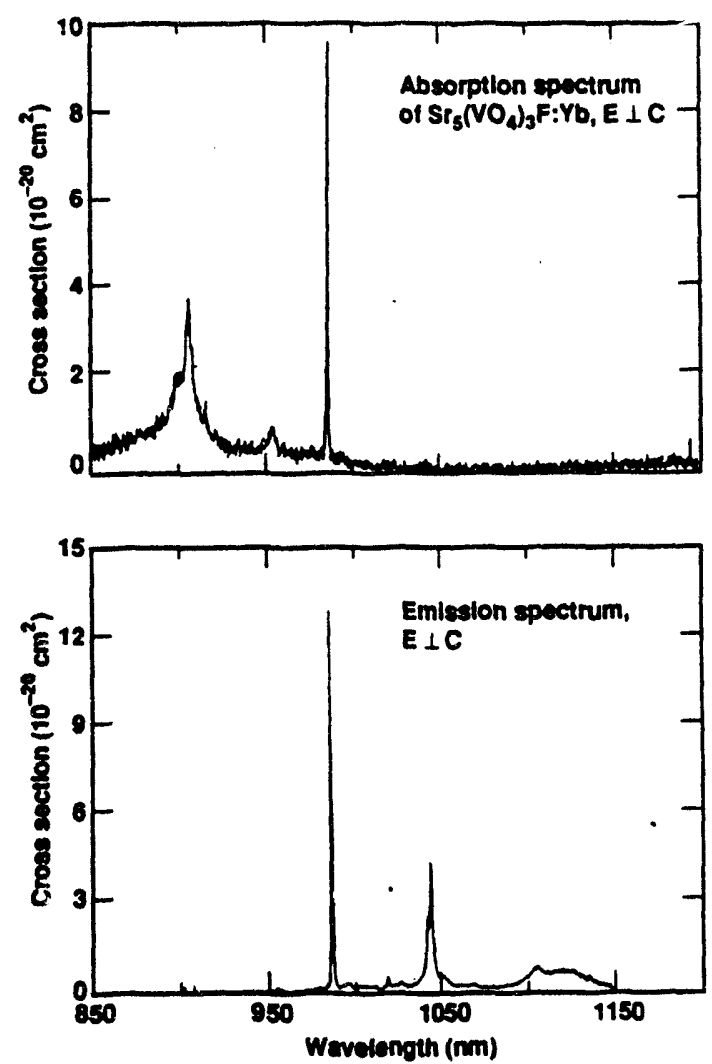

Figure 2. Absorption and emission spectra of $\mathrm{Yb}: \mathrm{Sr}_{5}\left(\mathrm{VO}_{4}\right)_{3} \mathrm{~F}$ with $\mathrm{E} \perp \mathrm{c}$.

The polarized absorption and emission cross sections of $\mathrm{Yb}$ :S-VAP are plotted for E\|c and $E \perp c$ in Figs. 1 and 2, respectively. Interestingly, the uniaxial nature of the apatitestructure has led to highly anisotropic, favorable spectral features. In particular, for light polarized along the c-axis of the crystal, the absorption spectrum is dominated by a single, strong feature at $905 \mathrm{~nm}$. Similarly, in the emission spectrum of E\|c, the oscillator strength is largely concentrated into the feature at $1044 \mathrm{~nm}$. The high values of $\sigma_{\mathrm{abs}}$ and $\sigma_{\mathrm{em}}$ observed for these two peaks suggest useful pump and extraction parameters for an $\mathrm{Yb}$ :S-VAP laser. We have previously observed similar useful attributes in crystals of $\mathrm{Yb}: \mathrm{FAP}^{5}$ and Yb:S-FAP. 6 More recently, spectral determinations have additionally been completed ${ }^{7}$ for several mixed crystals of

$\mathrm{Ca}_{5-x} \mathrm{Sr}_{\mathrm{x}}\left(\mathrm{PO}_{4}\right)_{3} \mathrm{~F}: \mathrm{Yb}$ where $\mathrm{x}=1,2$ and 3 (referred to as CS-FAP). As a useful way to compare the potential laser performance of known Yb-doped apatite crystals, we display data points on a figure-of-merit plot with $\sigma_{\mathrm{em}}$ on the ordinate and $I_{\min }$ on the abscissa. Such a plot is presented in Fig. 3 and includes the data points of several $\mathrm{Yb}$-doped crystals which we have presented in an earlier article. ${ }^{4}$ This plot very clearly demonstrates that the favorable high $\sigma_{e m}$, low $I_{\min }$ space is uniquely occupied by the various apatite structure crystals we have been investigating.

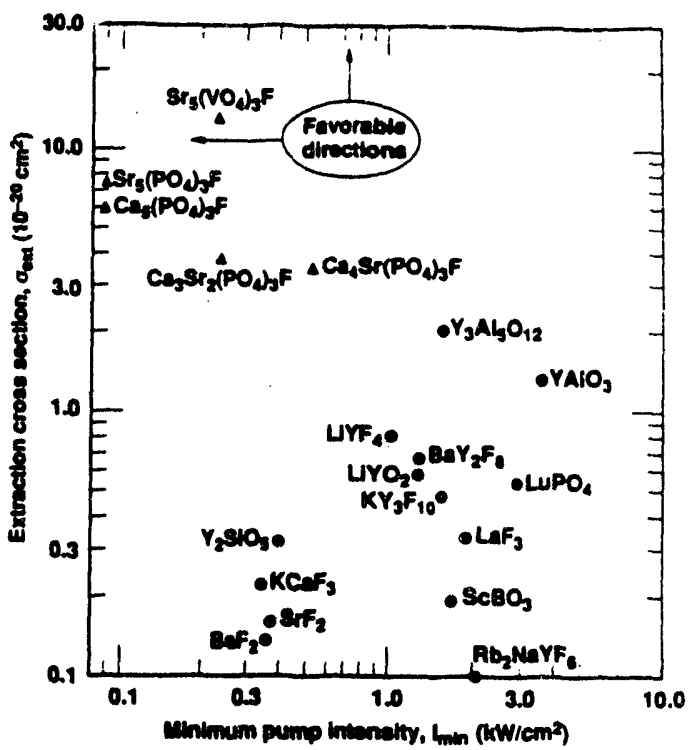

Figure 3. Plot of the extraction cross section ( $\sigma_{\text {ext }}$ ) against the minimum absorbed pump intensity required to yield net gain (Imin). All of the $\mathrm{Yb}$-doped apatite-structure crystals are located in the superior upper-left-hand sector of this plot.

\section{LASER PERFORMANCE}

The $\mathrm{cW}$ laser performance was assessed for all but one of the crystals that were spectroscopically characterized in the last section. Here we present data to prove that the Ca-Sr mixed apatite crystals, as well as the new fluorovanadate host, serve as useful laser media. Furthermore, the prior results obtained for Yb:FAP 5 and Yb:S-FAP6 will be briefly reviewed. Overall, four new Yb-based laser crystals have been recognized to lase effectively.

Since we have already described the techniques employed to measure the laser efficiencies, 5,6 only the highlights of our methods will be outlined here. The Yb-doped crystals were cut to $0.5-1.0 \mathrm{~cm}$ in length with parallel uncoated windows, and were arranged to be at the center of a $10 \mathrm{~cm}$ long concentric cavity. The Ti:sapphire pump laser output was 
set to $900-905 \mathrm{~nm}$, and chopped to a duty cycle of $25 \%$ before impinging on the crystal (although operating in the true $\mathrm{cw}$ mode appeared to have little or no impact on the $\mathrm{Yb}$ laser output). Using this experimental approach, we have produced laser action in Yb:S-VAP $\left(\mathrm{Sr} 5\left(\mathrm{VO}_{4}\right)_{3} \mathrm{~F}\right), \mathrm{Yb}: \mathrm{C}_{4} \mathrm{~S}-\mathrm{FAP}\left(\mathrm{Ca}_{4} \mathrm{Sr}_{2}\left(\mathrm{PO}_{4}\right)_{3} \mathrm{~F}\right)$, and $\mathrm{Yb}: \mathrm{C}_{3} \mathrm{~S}_{2}$-FAP $\left(\mathrm{Ca}_{3} \mathrm{Sr}_{2}\left(\mathrm{PO}_{4}\right)_{3} \mathrm{~F}\right)$. Several examples of the laser data oblained for Yb:SVAP are displayed in Fig. 4, where the output couplings employed ranged from $4.4 \%$ to $19.8 \%$, yielding slope efficiencies of 36.6, 56.4, and $62.9 \%$, with respect to the absorbed pump power. Several additional plots of the output power versus absorbed pump power appear in Fig. 5, including $\mathrm{Yb}$ in both of the mixed apatite hosts, $\mathrm{C}_{3} \mathrm{~S}_{2}-\mathrm{FAP}$ and $\mathrm{C}_{4} \mathrm{~S}-\mathrm{FAP}$, operating near the standard $1046 \mathrm{~nm}$ wavelength.

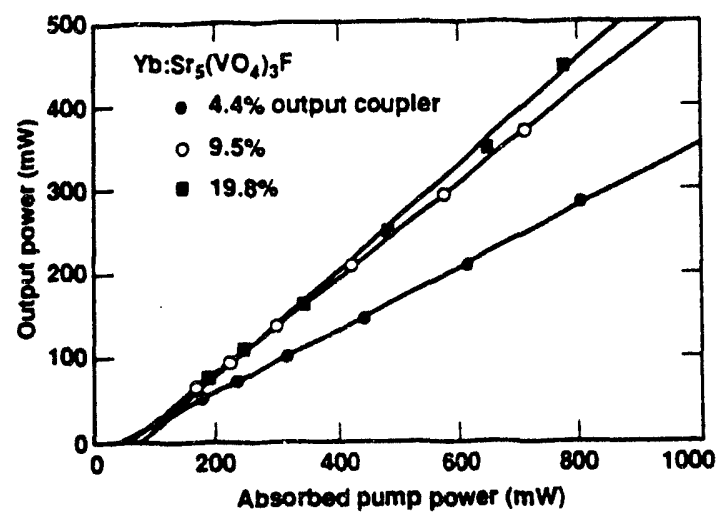

Figure 4. Laser efficiency data acquired for Yb:S-VAP for the three indicated output coupler values at $1044 \mathrm{~nm}$ with E\|c.

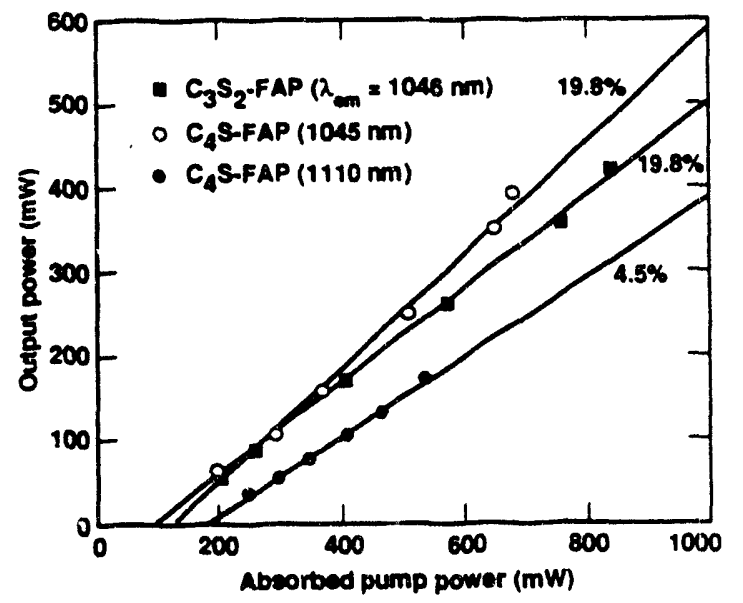

Figure 5. Illustrative laser efficiency results obtained for the mixed apatites, including $\mathrm{Yb}: \mathrm{C}_{3} \mathrm{~S}_{2}$-FAP at $1046 \mathrm{~nm}$, and $\mathrm{Yb}: \mathrm{C}_{4} \mathrm{~S}-\mathrm{FAP}$ at 1045, 1110, and $985 \mathrm{~nm}$ (output coupling values are denoted on the figure).
Interestingly, we were able to also lase the $\mathrm{Yb}: \mathrm{C}_{4}$ S-FAP crystal at $1110 \mathrm{~nm}$, which corresponds to a longer wavelength, weaker emission band. We expect that all of the $\mathrm{Yb}$ doped apatite crystals are amenable to laser action near this longer wavelength. It is noteworthy that, in spite of the low cross section value, the generation of $1110 \mathrm{~nm}$ output is somewhat enhanced by the fact that true fourlevel operation is possible at this wavelength (i.e., no ground state absorption is present). Note that $\mathrm{Yb}: \mathrm{C}_{4} \mathrm{~S}-\mathrm{FAP}$ has also been demonstrated to lase at $985 \mathrm{~nm}$ (with $\mathrm{E} \perp \mathrm{c}$ ). Since this spectral line corresponds to the transition between the lowest crystal field components of the ${ }^{2} \mathrm{~F}_{7} / 2$ ground and ${ }^{2} \mathrm{~F}_{5} / 2$ excited states, these experiments correspond to true three-level operation of this system. Therefore the 985,1046 and $1110 \mathrm{~nm}$ operating wavelengths of $\mathrm{Yb}: \mathrm{C}_{4} \mathrm{~S}-\mathrm{FAP}$ correspond to three, quasi-four, and four-level operation of the laser!

The laser data can be analyzed further on the basis of the simple equation describing the slope efficiency, $\eta$, in the limit of low (doublepass) loss, $L_{d}$, and low output coupling, $T$ :

$$
\eta=\eta_{0} \frac{T}{T+L}
$$

Here, $\eta_{0}$ provides a measure of the so-called intrinsic efficiency and is, in principle, simply given by the ratio of the pump and laser wavelengths (about $86 \%$ for the Yb:apatite crystal family). A summary of the single-pass losses and intrinsic efficiencies obtained for all of the Yb:apatite laser crystals is summarized in Table I, where the $\eta_{0}$ values of $65-84 \%$ are all in accord with the imposed fundamental quantum limit of $86 \%$ - the tendency to not attain the theoretical maximum is most likely a consequence of inadequate mode-matching of the pump and cavity fields. The range of singlepass losses from 0.7 to $3.0 \%$ is the result of variation in the quality of optical materials that have been employed. On the basis of the data presented in this section, the laser performance of the $\mathrm{Yb}$-doped apatite crystals is judged to be good at this time, and may be expected to improve further as lower loss material becomes available. 
Table I. Summary of laser efficiency analysis for $\mathrm{Yb}$-doped apatite crystals

$\begin{array}{lcccc}\text { Crystal } & \begin{array}{c}\text { Wavelength } \\ \text { (nm) }\end{array} & \begin{array}{c}\text { Intrinsic } \\ \text { efficiency (\%) }\end{array} & \begin{array}{c}\text { Single-pass } \\ \text { loss (\%) }\end{array} & \begin{array}{c}\text { Path } \\ \text { length (cm) }\end{array} \\ \text { S-VAP } & 1044 & 83 & 3.0 & 0.57 \\ \text { FAPa } & 1043 & 84 & 1.2 & 0.69 \\ \text { C4S-FAP } & 1046 & 76 & 1.7 & 0.75 \\ \text { C3S2-FAP } & 1110 & 81 & 2.7 & \\ \text { S-FAPb } & 1046 & 65 & 1.8 & 0.65 \\ & 1047 & 70 & 0.7 & 0.70\end{array}$

a Ref. 6

bef. 7

\section{CONCLUSION}

We have defined a new class of laser media based on the $\mathrm{Yb}^{3+}$ ion that offers a substantial range of laser parameters. The emission cross section was found to be as low as $3.6 \times 10^{-20} \mathrm{~cm}^{2}$ for $\mathrm{Yb}$ in the $\mathrm{Ca}_{4} \mathrm{Sr}\left(\mathrm{PO}_{4}\right)_{3} \mathrm{~F}\left(\mathrm{C}_{4} \mathrm{~S}-\mathrm{FAP}\right)$ host, and as large as $13.1 \times 10^{-20} \mathrm{~cm}^{2}$ for $\mathrm{Yb}: \mathrm{Sr}_{5}\left(\mathrm{VO}_{4}\right)_{3} \mathrm{~F}$ (S-VAP). The emission cross section variations are, of course, directly tied to changes in the emission lifetime (0.59-1.26 msec). All of the crystals have been demonstrated to lase efficiently and can be grown as high-quality optical materials by the Czochralski method. We believe that the Ybapatite class of lasers is useful for low and medium-power applications, and will be preferred over the more common $\mathrm{Nd}$-lasers in many situations by virtue of their longer lifetime.

\section{ACKNOWLEDGMENTS}

We wish to thank John Tassano and Wayne Kway for providing many of the crystals used in this study. This work was performed under the auspices of the U.S. Department of Energy by Lawrence Livermore National Laboratory under contract no. W-7405-ENG-48.

\section{REFERENCES}

1. G. A. Bogomolova, D. N. Vylegzhanin, and A. A. Kaminskii, Sov. Phys.JETP 42,440 (1976).

2. A. R. Reinberg, L. A. Riseberg, R. M. Brown, R. W. Wacker, and W. C. Holton, Appl. Phys. Lett. i9 11 (1971).

3. W. F. Krupke and L. L. Chase, Opt. Quantum Electron. 22, S1 (1990).

4. L. D. DeLoach, S. A. Payne, L. L. Chase, L. K. Smith, W. L. Kway, and W. F. Krupke, J. Quantum Electron.29 1179 (1993).

5. S. A. Payne, L. K. Smith, L. D. DeLoach, W. L. Kway, J. B. Tassano, W. F. Krupke, IEEE "Laser, optical, and thermomechanical properties of $\mathrm{Yb}$ doped fluorapatite," IEEE J. Quantum Electron. 30 (1), 1994.

6. L. D. DeLoach, S. A. Payne, L. K. Smith, W. L. Kway, and W. F. Krupke, "Laser and spectroscopic properties of $\mathrm{Sr}_{5}\left(\mathrm{PO}_{4}\right)_{3} \mathrm{~F}: \mathrm{Yb}$," to be published in J. Opt. Soc. Am. B.

7. L. D. DeLoach, S. A. Payne, B. H. T. Chai, W. F. Krupke, L. K. Smith, W. L. Kway, and ]. B. Tassano, "Laser and spectroscopic properties of Yb-doped apatite crystals," in the OSA Proceedings on Advanced Solid State Lasers, vol. 15, eds. A. A. Pinto and T. Y. Fan (Optical Society of America, Washington, DC, 1993), p. 188.

8. B. H. T. Chai, G. Loutts, X. X. Zhang, S. A. Payne, W. F. Krupke, L. D. Deloach, and L. K. Smith, to be published.

9. R. C. Ohlmann, K. B. Steinbruegge, and R. Mazelsky, App. Opt. Z 905 (1968).

10. P. Lacovara, H. K. Choi, C. A. Wang, R. L. Aggarwal, and T. Y. Fan, Opt. Lett. 6, 1089 (1991). 

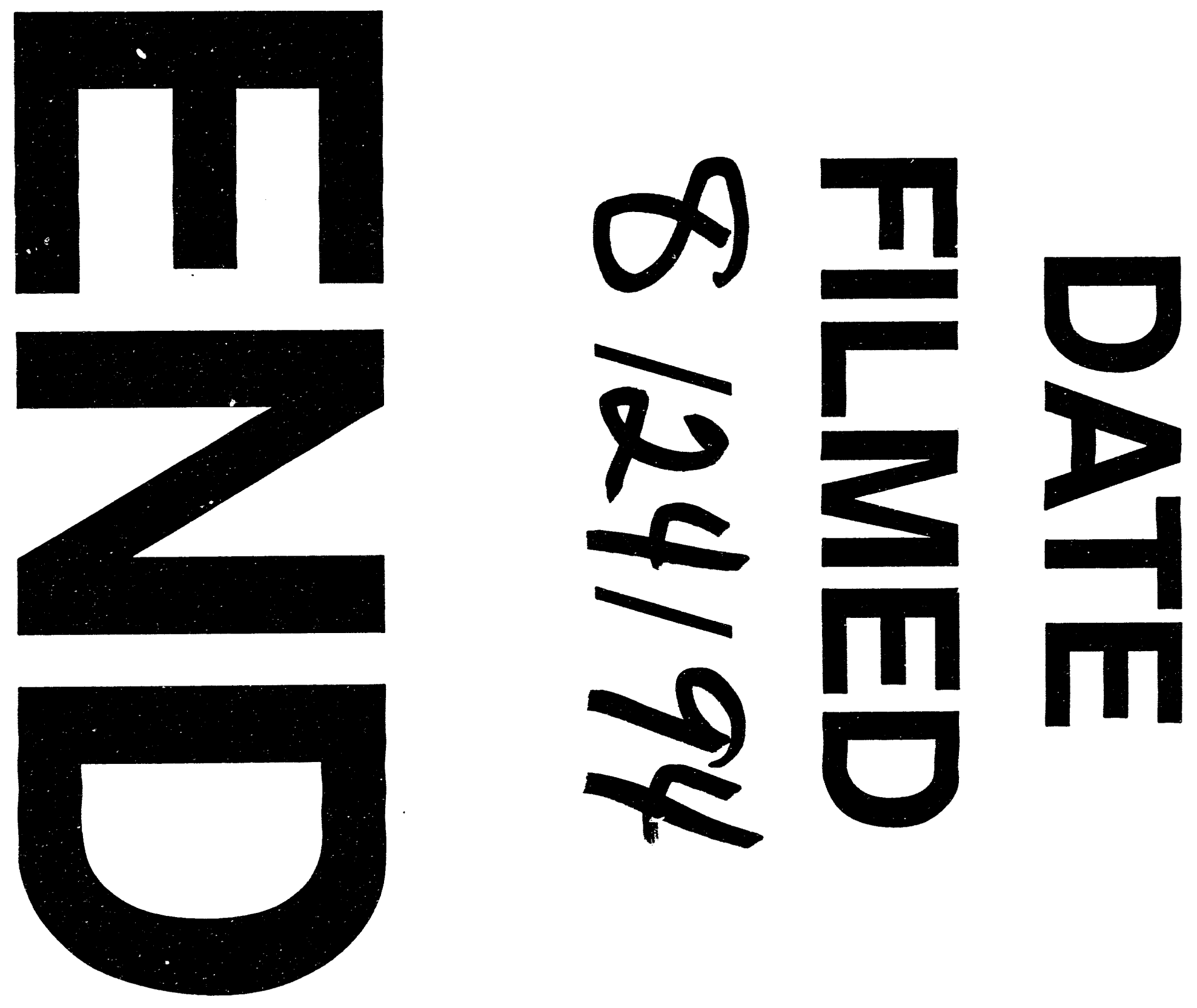
